\title{
Análisis de la experiencia docente en clases de Educación Física durante el confinamiento por COVID-19 en México Analysis of teaching experience in Physical Education classes during COVID-19 confinement in Mexico

\author{
Ramón Alfonso González-Rivas, Gabriel Gastélum-Cuadras, W ilberth Velducea Velducea, Javier Bernabé \\ González Bustos, Susana D omínguez Esparza \\ UniversidadAutónomadeChihuahua(México)
}

\begin{abstract}
Resumen. El objetivo deestainvestigación fueanalizar laexperienciadocentedel profesorado deEducación Físicaen susclasesdurante el confinamiento por COVID-19 en M éxico. Esun estudio descriptivo, transversal, con enfoquemixto; participaron 79 profesoresde Educación Físicaen escuelaspúblicasdeEducación Básica, del Estado deChihuahua, México; 52 hombresy 27 mujeres $\left(M_{\text {edal }}=40.3, D T=\right.$ 8.2); con experiencia docente ( $\left.M_{\text {años }}=14.1, D T=7.6\right)$, el $12.7 \%$ impartió clase en nivel preescolar, $83.5 \%$ en primariay $3.8 \%$ en secundaria, el $60.8 \%$ en escuelasubicadas en lazonaurbanay $39.2 \%$ en zonarural. Seanalizaron lasvariables deestrategiasdidácticas, evaluación deaprendizajes, resilienciadocente, afectacionesdel confinamiento por COVID-19 en losalumnosy capacitaciones requeridas por los docentes; paratal efecto, sediseñó y aplicó un instrumento ad hoc. Losresultados señalan quelamayoríadel profesorado utilizó lametodologíadeaulainvertidaeimpartió clases de maneravirtual, laevaluación fueun reto complejo, desarrollaron actitudesy valores detoleranciay empatía, así como habilidadesenTIC, identificaron la posibilidad de aparición de problemas psicológicosen susalumnos, al gunos profesores mencionaron la necesidad de capacitaciones en temas de pedagogía,TIC y recomendaciones de actuación en el regreso aclases presenciales. Se concluyó queel profesorado deEducación Físicaenfrentó diversosretosen su clase; sin embargo, la resilienciadocentemanifiestaen paciencia, empatíay desar rollo de habilidadesenTIC les permitieron desempeñar su trabajo.
\end{abstract}

Palabrasclave: Educación Física, COVID-19,TIC, Estrategiasdidácticas, Evaluación de aprendizajes.

\begin{abstract}
The aim of this research was to analyze the teaching experience of Physical Education teachers in their classes during COVID-19 confinement in M exico. Thisisadescriptive and cross-sectional study with amixed approach; participantswere 79 Physical Education teachersat public elementary schools, in theState of Chihuahua, M exico; 52 males and 27 females $\left(M_{2 a e}=40.3, S D=8.2\right)$; W ith teaching experiences $\left(M_{\text {yeas }}=14.1, S D=7.6\right), 12.7 \%$ at preschool level, $83.5 \%$ at primary, $3.8 \%$ at secondary, $60.8 \%$ in schools located in urban areas, and $39.2 \%$ in rural areas. The variables of didactic strategies, learning assessment, teacher resilience, effects of COVID-19 confinement on the students, and training required by teacherswereanalyzed; For this purpose, an ad hoc instrument was designed and applied, which consists of 13 items. Theresultsindicated that most teachersused theflipped classroom methodology and taught virtual classes, the eval uation was complex and challenging, however, they developed attitudes and values of tolerance and empathy, as well asskills in ICT, they were al so able to identify the possibilities of psychological problems appeared in their students, some teachersmentioned the need for training in pedagogy, ICT and recommendations for action when returning to face-to-face classes. It was concluded that Physical Education teachersarefaced with various challenges in their class; however, teachers' resilience manifested with patience, empathy, and the development of ICT skillsall owed them to carry out their work.
\end{abstract}

Keywords: Physical Education, COVID-19, ICT, Didactic strategies, Learning assessment.

\section{Introducción}

El 31 de diciembre del 2019, las autoridades de la ciudad de Wuhan en China reportó un conglomerado de casos de neumonía, a pesar de los esfuerzos realiza dos por el país y la Organización Mundial de la Salud (O MS) fue imposible contener el virus por COVID-19, se declaró pandemia el 11 de marzo del 2020 (O MS, 2021).

La pandemia impactó fuertemente a la población

Fecha recepción: 23-11-20. Fecha de aceptación: 26-02-21

Gabriel Gastélum Cuadras

gastelum@ uach.mx mundial en todos los aspectos, principalmente el sanitario, pero no se puede dejar de lado las afectaciones en el sistema educativo en todos niveles (Wang, Zhang, Zhao, Zhang \& Jiang, 2020). Con la finalidad de controlar el virus, el distanciamiento social fue la primera medida preventiva (Adhikari et al., 2020), lo que limitó la práctica de actividad física en sus diversas manifestaciones (Blocken, Malizia, Druenen \& Marchal, 2020).

El confinamiento por COVID-19 modificó la intervención del profesorado de Educación Física (EF) con sus alumnos, encontrando problemas pedagógicos y didácticos (Baena-Morales, López-M orales \& GarcíaTaibo, 2020); debido a la natural eza de la asignatura(tipo de actividades, necesidades de espacio, interacción, 
movimiento, entre otras), el profesorado enfrentó un reto complejo, fue necesario generar procedimientos específicos que aseguraran el bienestar durante la clase, tanto de estudiantes como profesores (COLEF, 2020). Ante esta situación el profesorado de EF tuvo la necesidad de adaptar sus clases, surgiendo diferentes propuestas pedagógicas, sin perder de vista la adquisición de competencias, evaluación y utilización de recursos de tecnologías de información y comunicación (TIC) (Burgueño et al., 2021; Burgueño, Espejo, LópezFernández \& Gil-Espinosa, 2020; Hall \& 0 choaMartínez, 2020).

Desde hace al gunos años, las TIC han sido tendencia en la actividad física debido a las posibilidades pedagógicasinnovadorasque representan (Burgueño et al., 2021; Carriedo, M éndez-Giménez, Fernández-Río \& Cecchini, 2020; Fernández, M ontenegro, Fernández \& Tadeu, 2021; Gómez, Rodríguez \& Ramos, 2019; Gómez-Gonzalvo, M olina-Alventosa \& Devís, 2018); su aplicación suele despertar el interés de los estudiantes, por lo que es conveniente en el contexto de la EF (Aznar, Cáceres, Trujillo \& Romero, 2019; Moreno, Rodríguez, Navas-Parejo \& Sola, 2020; M elnyk, 2017; Piedra, 2020).

La pandemia dejó al descubier to la falta de conciencia social respecto a la importancia de la actividad física, el descuido sistemático y el poco valor curricular hacia la asignatura de EF en escuelas públicas (Pinasa, 2020). A pesar de la pandemia, las clases de EF son fundamentales, niños, niñas y adolescentes no deben descuidar la actividad física, pero es imperativo atender las recomendaciones de salud (Kalazich et al., 2020). Se sugiere realizar actividad física en casa, y hasta que exista bajo riesgo de contagio, se debe atender los protocolos de salud establecidos (Cossio-Bolaños, 2020; Chen et al. , 2020a; Dixit, 2020).

La EF es un derecho fundamental que favorece el desarrollo integral de individuos, familias, comunidad y sociedad; de acuerdo con la O rganización de las N aciones Unidas para la Educación, la Ciencia y la Cultura (2015) estimula el hábito de la práctica de actividad física favoreciendo la calidad de vida de las personas, teniendo injerencia en el desarrollo motriz, fisiológico, psicológico, cognitivo, rendimiento académico y promoción de valores personales y sociales; por lo que se sugiere horas de calidad de EF (Betancourt, Bernate, Fonseca\& Rodríguez, 2020; Giakoni, Paredes\& DuclosBastías, 2021; Guillamón, García \& Martínez, 2021; Guillamón, García\& Martínez, 2020; Guthold, Stevens, Riley \& Bull, 2020; Rodríguez, Valenzuela \& Martínez,
2016).

El rol del profesor es fundamental, cuando permite que los estudiantes sean proactivos y tengan libertad de tomar decisiones se logra un mayor disfrute de la actividad impactando en la autoestima (Baños $\&$ Arrayales, 2020; Hernández, González, Sánchez \& Carrión, 2020; Salazar \& Gastélum, 2020; Soares et al., 2021; Zueck, Ramírez, Rodríguez e Irigoyen, 2020). Por ende, es trascendental que el profesor diseñe y aplique activida des innovadoras, desafiantes y atractivas, en una atmósfera adecuada donde los estudiantes puedan real izar actividad física en un ambiente lúdico y seguro, favoreciendo competencias motrices, sociales y emocionales (García, Sánchez \& Ferriz, 2021; M uñoz-Arroyave, Lavega-Burgués, Costes, Damian \& Serna, 2020), Iogrando que el estudiante genere hábitos de vida saluda bles, por medio de laactividad física(Bañoset al., 2019), sin embargo, la pandemia modificó el accionar de los profesores de EF (Baena-Morales et al., 2020).

En México, más de 33 millones de estudiantes de Educación Básica (EB) fueron afectados debido a que durante el confinamiento las escuelas cerraron sus puertas (UNESCO, 2020), el 23 de marzo de 2020 la Secretaria de Educación Pública (SEP) suspendió indefinida mente las clases presenciales a nivel de EB, dando lugar a la educación a distancia (SEP, 2020). La escuela es el espacio ideal para generar conductas saludables (Bogantes, M onge, González, Víquez \& Vargas, 2020) y en este país, representa el único espacio en el que los niños, niñas y adolescentes realizan ejercicio físico obligatoriamente (Zueck et al., 2020). El confinamiento provocó sedentarismo, miedo, estrés, ansiedad en la población (Celis-M orales, Salas-Bravo, Yáñez \& CastiIlo, 2020; Chen et al. , 2020a; Chen et al. , 2020b; Islam, Bodrud-D oza, K han, Haque \& Mamun, 2020; Shechter et al., 2020), como se leído en líneas anteriores, los beneficios de la EF pueden disminuir las afectaciones psicológicas y fisiológicas de la pandemia; sin embargo, durante el confinamiento el profesorado enfrentó diversos retos para realizar su trabajo, teniendo que adaptarse a las recomendaciones de salud (Baena-M oral es et al., 2020). De esta manera, el objetivo de este estudio fue analizar la experiencia docente del profesorado de EF en sus clases durante el confinamiento por COVID19 en México.

\section{M etodología}

\section{Participantes}

Se envió el instrumento a 103 profesores (elegidos 
al azar) de $E F$ en $E B$, respondiendo una muestra no probabilística de 79 (tasa de respuesta 76.6\%); 52 hombres y 27 mujeres $\left(M_{\text {edad }}=40.3, D T=8.2\right)$; con experiencia docente ( $M=14.1$ años, $D T=7.6)$. El $12.7 \% \mathrm{im}$ partió clases en preescolar, $83.5 \%$ en primaria y $3.8 \%$ en secundaria. En cuanto alaubicación geográfica, $60.8 \%$ trabajó en escuelas en la zona urbana y $39.2 \%$ en zona rural. Los criterios de inclusión fueron: profesores de EF que impartieran clase en EB durante el periodo de confinamiento en escuelas públicas, en cualquier nivel educativo (preescolar, primaria y secundaria), en este estudio no se contempló al profesorado de escuelas particulares.

\section{Diseño}

Este estudio tiene un diseño descriptivo, transversal, con enfoque mixto de tipo Cual-cuan, que permitió analizar fenómenos dando mayor peso al anál isis cualitativo sobre el cuantitativo (Hernández, Fernández \& Baptista, 2014). Este estudio anal izó cómo la suspensión de clases presenciales en EB a consecuencia de la pandemia por COVID-19, dando lugar a la educación a distancia; influyó en las clases de EF desde las perspectivas de:

Estrategias didácticas: entendidas como metodologías que utiliza el profesor para que los estudiantes logren adquirir el conocimiento y desarrollar competencias (O rozco, 2016).

Evaluación de aprendizajes: es el proceso sistemático que ofrece información sobre el cumplimiento de metas académicas, emitir juicios y toma decisiones respecto a la formación de los estudiantes (Contreras, 2010).

Resiliencia docente: es la aptitud de adaptarse de buena forma ante una situación compleja a fin de desa rrollar competencias sociales, académicas y vocaciona les pese eventos adversos ( $G$ ardner \& Stephens-Pisecco, 2019).

Afectaciones del confinamiento por COVID-19 en los alumnos: son las posibles afectaciones de los alumnos durante el confinamiento, desde la perspectiva del profesorado.

Capacitaciones requeridas por el profesorado: son los cursos, conocimientos y contenidos de interés del profesorado para desempeñar su trabajo en el contexto de la pandemia por COVID-19.

Laoperacionalización de estas variablesfuepor medio de cada una de las categorías preestablecidas en el instrumento. Este estudio se desarrolló bajo los lineamientos de la Declaración de Helsinki (2015).

\section{Instrumentos y herramientas}

En la revisión bibliográfica, no se encontró un cuestionario con las características que se requerían para esta investigación, por lo que se diseñó un instrumento ad hoc, el «Cuestionario experiencias docentes del profesorado de EF durante el confinamiento por COVID19 «(anexo); su validación fue por medio de la metodología de jueces expertos, tomando nociones de Escobar-Pérez y Cuervo-M artínez (2008). En primera instancia se diseñó el cuestionario que contempló cinco categorías preestablecidas: 1) estrategias didácticas, 2) evaluación de aprendizajes, 3) resiliencia docente, 4) afectaciones del confinamiento por COVID-19 en los alumnos y 5) capacitaciones requeridas por los docentes; se integró un grupo de tres expertos en investigación educativa, pedagogía en EF y con experiencia impartiendo clases de EF en EB, mismos a quienes se les envió el cuestionario para conocer sus sugerencias y opiniones.

Una vez recibida la retroalimentación se realizaron las modificaciones pertinentes y se envió de nuevo a revisión con los tres jueces expertos; en esta nueva etapa, fue necesario comprobar el grado de concordancia de las valoraciones de los jueces, se utilizó el análisis estadístico deW de Kendall, obteniendo valores aceptados de .825 en suficiencia, .762 en coherencia, .762 en relevancia y .796 en claridad de los ítems, valores que dan validez y confiabilidad al instrumento. A continuación, se describe la experiencia de los jueces expertos:

Juez 1.- docente universitario e investigador con 10 años de experiencia en investigación educativa, miembro del sistema nacional de investigadores, con diversos artículos publicados en revistas internaciones.

Juez 2.- docente universitario e investigador experto en pedagogía de la EF, con publicaciones internacionales, con 5 años de experiencia como profesor de EF en EB en nivel preescolar.

Juez 3.- docente universitario e investigador con publicaciones internacionales y 13 años fungiendo como profesor de EF en EB en nivel primaria.

\section{Análisis de datos}

Se realizaron los análisis estadísticos descriptivo, frecuencias yW de Kendall (para la validación del instrumento), para lo cual se utilizó el SPSS versión 22. En cuento al análisis cualitativo, se utilizó el diseño emergente de Glasser (1992) para codificar e interpretar cada una de las cinco categorías preestablecidas en el instrumento y que corresponden a las variables del es- 
tudio, se utilizó el programaAtlas ti versión 7.5.

\section{Procedimiento}

En primera instancia se diseñó el instrumento para la recogida de datos. El cuestionario se añadió a la aplicación Google formularios; entre los días del 4 al 18 de octubre del 2020 se envió el enlace de acceso a 103 docentes de EB que impartieron la asignatura de EF en Chihuahua, M éxico. El cuestionario fue respondido por $79(76.6 \%)$, con participación voluntaria y garantizando el anonimato.

A partir del día 19 de octubre se realizó el análisis estadístico descriptivo de los 79 profesores que contestaron el cuestionario, se obtuvieron los porcentajes de las preguntas con respuesta dicotómica, para tal efecto se utilizó el software SPSS. Para el análisis de las preguntascon respuesta abierta, se creó un documento RTF con los comentarios del profesorado; previo al análisis, dos de los investigadores unificaron criterios pararealizar de manera independiente el proceso de codificación e interpretación de cada una de las cinco categorías preestablecidas en el cuestionario, posteriormente se compararon los resultados de ambos investigadores para tener un producto final.

\section{Resultados}

\section{Categoría de estrategias didácticas}

El 54.4\% del profesorado que participó en este estudio mencionó haber cumplido con el programa de EF en EB, en base a los comentarios de los profesores. Se identificó la utilización del modelo de EF: autoconstrucción de materiales, apoyándose en el cua dernillo didáctico y en los aprendizajes esperados. Se encontró que utilizaron las metodologías de aula invertida, aprendizaje cooperativo (con apoyo de padres, madres o tutores legales) y aprendizaje basado en problemas; para tal efecto, fue indispensable el uso deTIC como videollamadas, elaboración de videos y plataformas educativas gratuitas como Google clasroom, tres profesores mencionaron haber enviado actividades impre- sas a los estudiantes. El apoyo de los padres, madres 0 tutores legales fue mencionado en dos aspectos: como medio de comunicación para el envío y apoyo en la rea lización de las actividades; en este sentido, algunos profesores expresaron haber tenido que adaptar sus planificaciones al contexto de los estudiantes.

«Adaptación de juegos recreativos tradicionales para jugar en espacios reducidos... los entrego como archivo PDF vía WhatsApp y cuadernillo impreso».

«Comunicación con los papás por medio deW hatsApp, enviar actividades sencillas con poco material y complejidad, envío videos realizando las actividades como ejemplo para los alumnos y tutoresy 
"Al final de las actividades envío un cuadro que deben contestar los papás donde responden sí el niño pudo realizar la actividad o las actividades sin dificultades».

«Recopilo vídeos de las evidencias de mis alumnos, en ellos puedo hacer uso de la observación para medir si sus avances están en función del aprendizaje esperado». para los estudiantes. Ú nicamente cinco profesores expresaron que fue aceptable, tres no evaluaron los aprendizajes de sus estudiantes.

«M uy complicada porque muy pocos al umnos están respondiendo, y no podemos reprobar a los que no responden porque en realidad no sabemos las causas exactas por las que no están trabajando. Por otrolado, el hecho de no reprobarl os no sería justo para los que si respondieron y estuvieron trabajando en medida desus posibilidades».

«Es un tanto incierta puesto que no podemos estar viendo el proceso de los niños y tenemos que confiar en las percepciones de lo que ven los papás en sus hijos».

Figura 2. Evaluación de aprendizajes.

La mayoría de los profesores que participaron en esta investigación mencionaron que la evaluación fue irreal y difícil, otros la refirieron como mala, insuficiente y que representó un retroceso académico; destacó que cuatro profesores la catal ogaron como estresante

\section{resiliencia docente}

\section{Categoría}

de
El $67.1 \%$ del profesorado mencionó haber tenido dificultades para desempeñar su trabajo, específicamente en referencia a las estrategias didácticas $31.6 \%$ expresó sentir falta de compromiso ya sea de padres, madres, tutores legales y/ 0 estudiantes, no poder dar retroalimentación en el momento de realizar la actividad motriz; problemas que se relacionan con falta de comunicación; en este sentido, lasfallas deconexión a internet pudieran explicar dichos inconvenientes. La falta de dominio de TIC fue otro inconveniente mencionado en menor medida. Destacó que cinco profesores mencionaron sentir sobrecarga de actividadesprofesionalesy familiares.

«No hay acceso y/ 0 muy malo el internet, el distanciamiento de las localidades, la falta decoordina-

Figura 4. Problemas identificados por el profesorado. 
ción y comunicación con los padres de familia-niños-maestro».

"Las actividades requieren apoyo de sus padres, cosa que no todos tienen, además de la falta de tecnología o recursos en su hogar».

De acuerdo con el profesorado de EF de este estudió, la paciencia y la empatía fueron los dos valores y actitudes desarrollados más citados; sin embargo, el respeto, responsabilidad, tolerancia y optimismo fueron mencionados en más de una ocasión. Más de la mitad de los docentes expresaron haber desarrollado habilidades en TIC y trabajo colaborativo con otros docentes. Destacó que 12 profesores mencionaron haber desarrollado adaptabilidad, relacionada con las capacida des de creatividad y resiliencia.

"He tenido que adecuarmeal manejo delas TIC para poder diseñar las actividades en digital para su posterior entrega y re cepción de evidencias. He tenido que ser más empático con aquellos niños que se les ha dificultado el trabajo a distancia por razones externas a ellos》.

«La utilización efectiva de las aplicaciones disponibles para lograr un mayor alcance en los alumnos, actitud positiva y siempre esperando la respuesta positiva de alumnos y padres, con los valores de trabajar en familia y estando al pendiente de cómo están, comunicación respetuosa y de empatía».

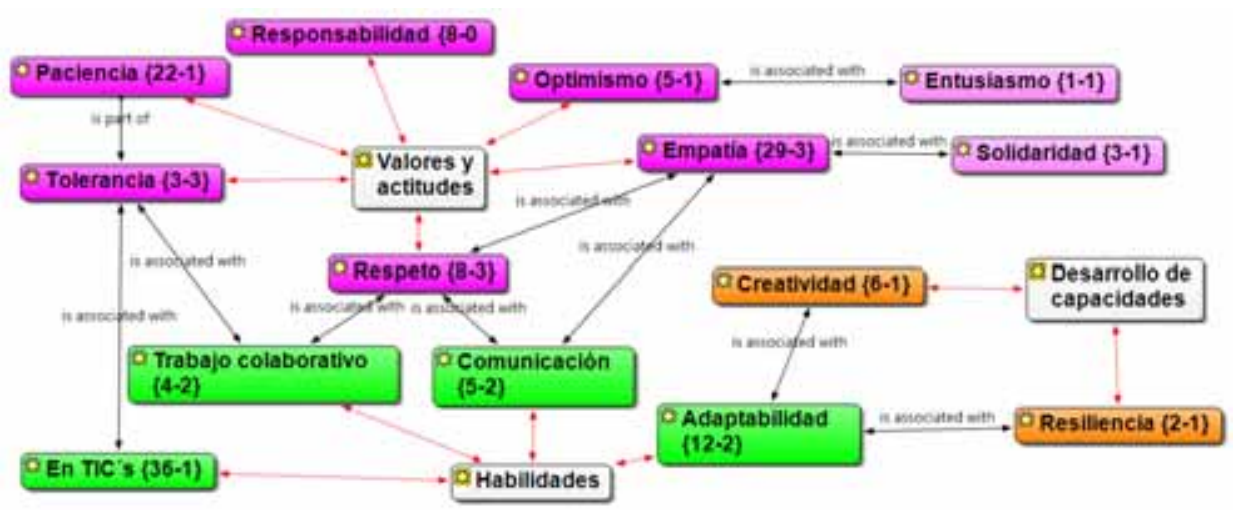

Figura 5. Actitudes, valores y habilidades desarrolladas por el profesorado. nerando estrés, depresión y ansiedad. Algunos comentarios revelan que el impacto psicológico pudiera repercutir en malos hábitos alimenticios y sedentarismo, generando obesidad y sobrepeso, repercutiendo en la condición física de los alumnos. Por último, relaciona ron las afectaciones psicológicas con aspectos sociales como la mala conducta y la disminución de socialización y la posibilidad de ser víctimas de violencia.

«Salud mental, ansiedad, estrés, también física por el problema de sobrepeso, por el tanto tiempo sin realizar actividad física».

«El confinamiento los está haciendo más pasivos y adquiriendo hábitos menos saludables como dormir y comer más».

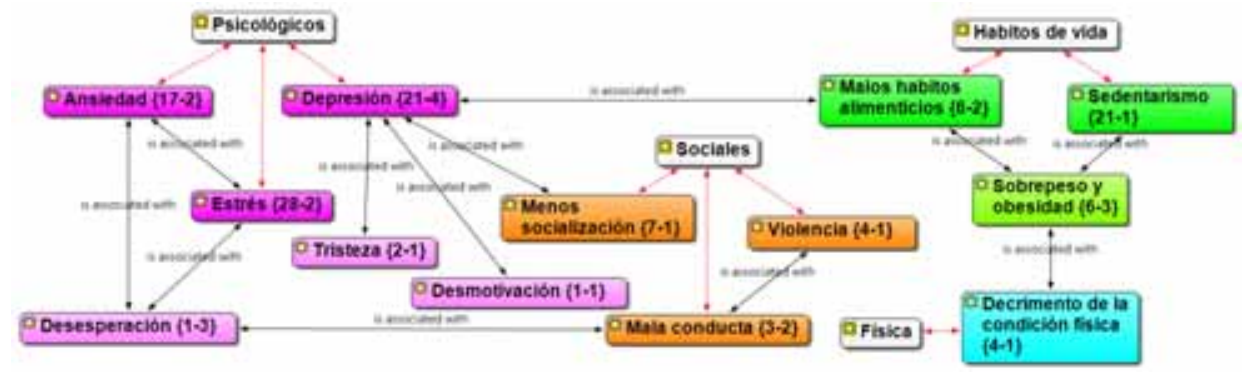

Figura 6. Afectaciones en los estudiantes, según el profesorado.

\section{Categoría de capacitaciones requeridas por los docentes}

El $22.8 \%$ del profesorado expresó no haber tenido algún tipo de capacitación, sugirieron la formación en pedagogía utilizando TIC y utilización de materiales reciclados en EF. En menor medida mencionaron la necesidad de aprender a tra bajar con los padres, madres y tutores legales en el esquema de educación virtual. Por último, expresaron el deseo de capacitaciones en seguridad, con tópicos relacionados a la higiene, autocuidado, cuidado de los demás y a las medidas a tomar en cuenta en el eventual regreso a clases presen-

\section{Categoría de afectaciones del confinamiento por COVID-19 en los alumnos}

El $86.1 \%$ del profesorado coincidió en que el confinamiento pudo afectar a sus estudiantes de diversas maneras, principalmente en aspectos psicológicos ge- ciales.

«Una capacitación de cómo enfrentar el problema para cuando regresemos qué medidas tomar, qué materiales usar si los que hay en la escuela o encargarlea cada alumno de su casa que valla desinfectado»

«Pues de cómo hacer para llegar a los alumnos, sin que los 
padres de familias se molesten porque ya estamos Ilegando a su intimidad del hogar». basadas solo en la experiencia.

Se encontró que el profesorado de EF adaptó sus clases al contexto de los estudiantes, con la fina lidad de alcanzar el aprendizaje esperado, en concordancia con Martínez-Hita (2020). En esta misma línea, paraFuentes (2020), las actividades deEF durante el confinamiento deberían generar libertad de movimiento, auto-

Figura 7. Capacitaciones requeridas, según el profesorado.

\section{Discusión}

El objetivo de este estudio fue analizar la experiencia docente del profesorado de EF en sus clases durante el confinamiento por COVID-19 en M éxico. Por lo que su importancia radica en identificar las situaciones que enfrentaron los profesores para desempeñar su trabajo.

Los resultados de este estudio señalan que la mayoría del profesorado que participó en este estudio impartió clases utilizando el modelo de autoconstrucción de materiales, consiste en la práctica de actividades físicas utilizando material es elaborados por el mismo estudiante (Fernández-Río, Calderón, Alcalá, Pérez-Pueyo $\&$ Cebamanos, 2016). Las metodologías de aula invertida y el aprendizaje cooperativo también fueron identificadas, ambashan demostrado ser eficacesen laEF (Bermejo et al., 2021; Fernández-Río, 2017; Recio, 2017). Se encontró que el profesorado impartió clases utilizando TIC; en esta misma línea se sitúan los resultados de Baena-M orales et al. (2020). De acuerdo con Piedra (2020), las redes sociales han sido valiosas para la implementación en clases de EF durante el periodo de confinamiento. LasTIC tienen un potencial transforma dor en el ámbito de la EF, por tal motivo los profesores deben reflexionar sobre su importancia (Carriedo et al. , 2020; Díaz, 2020). Para que los docentes pudieran impartir sus clases virtuales tuvieron que desarrollar competencias en el manejo de TIC, evidenciando actitudes positivas hacia su utilización; hallazgos similares a los de Barahona, García y Pañego (2020). Estos resultados manifiestan una inclinación hacia el uso deTIC en las clases de EF, aunque, se coincide con Pérez y Hortigüela (2020), quienes consideran que los docentes requieren lograr un equilibrio entre las tendencias vanguardistas como el uso de las redes sociales y clases nomía, ejercicio aeróbico, así como canalizar el desarrollo de habilidades motoras básicas y complejas. En este sentido, es fundamental adaptar e innovar practicas lúdicas en espaciosfuera de laescuelatradicional (García, 2020). El profesor de EF se ha visto en la necesidad de modificar sus recursos didácticos, para impactar de la mejor manera a sus alumnos; por tal motivo, debe ser considerado indispensable durante el aislamiento, ya que su labor contribuye al mejoramiento y mantenimiento de la salud (SilvaFilho et al., 2020).

En este estudio, la mayoría del profesorado expresó que la evaluación de manera virtual fue un reto complejo. Por lo que, de hecho, algunos expresaron que no la realizaron, resultados similares a los reportados por Baena-M orales et al. (2020). La concordancia en estos resultados sugiere que la evaluación de forma virtual es área de oportunidad de mejora paralos docentes. Hubo profesores que utilizaron estrategias de autoevaluación y coevaluación, lo que sin duda implicó al alumno en su proceso de aprendizaje, al igual que en el estudio de Barrientos, López y Pérez-Brunicardi (2019); al respecto, Canales-Núñez, Aravena, Carcamo-0 yarzún, Lorca y Martínez-Salazar (2018) consideran que en EF es conveniente involucrar a los estudiantes en el proceso de evaluación. En este estudio, al gunos profesores utiliza ron el portafolio de evidencias y diarios, hallazgos que desde la perspectiva de Calatayud (2019) significa un avance hacia la evaluación auténtica en laEF y por ende mejor formación en hábitos de vida saludables.

El profesorado de EF de la presente investigación encontró diversos inconvenientes para realizar su trabajo, problemas de conexión a internet, falta de comunicación con padres, madres o tutores legales y alumnos, sobrecarga de actividades; sin embargo, desarroIlaron actitudes, val ores y habilidades que les permitieron impartir sus clases pese a las diferentes problemá- 
ticas. En el contexto educativo la resiliencia es la capa cidad que permite analizar inconvenientes desde un enfoque que permite encontrar soluciones innovadoras de intervención y es fundamental para generar climas de aprendizaje positivos a pesar de la adversidad (Román et al., 2020; Vera \& Gabari, 2019). En este sentido, un buen profesor de EF se caracteriza por su implicación profesional que se manifiesta por medio de una buena planificación, intervención, evaluación, personalidad y el fomento de valores, pese a las circunstancias ( $D a$ Costa, 2006; Villaverde-Caramés, Fernández-Villarino, Toja-Reboredo \& González-Valeiro, 2021).

Desdelaperspectivadel profesorado, el confinamiento podría afectar la salud de los alumnos en aspectos psicológicos, sociales, físicos y en el desarrollo de malos hábitos de vida, hallazgos que se sitúan en la misma línea que los manifestados por Baena-Morales et al. (2020). En este estudio el profesorado expresó que sus al umnos fueron vulnerables a estrés, depresión y/ 0 ansiedad. El brote de COVID-19 generó miedo y estrés en diferentes países y se manifestó en problemas de sueño, mal humor y problemas familiares; el encierro sumado a un contexto socioeconómico desfavorecido puede causar ansiedad (Chen et al., 2020a; Islam et al., 2020; Shechter et al. , 2020). Pues, el síndrome deestrés COVID-19 es un fenómeno complejo que puede incluir diversos tipos de miedo (Taylor et al., 2020). Los docentes identificaron la posibilidad del desarrollo de há bitos de vida sedentarios en sus estudiantes, situación que concuerda con lo reportado por Chen et al. (2020b), después de meses de encierro se puede anticipar situa ciones desfavorables, los profesores de EF deben redoblar esfuerzos en la promoción de hábitos de vida saludables, con la finalidad prevenir otro problema sanita rio como lo es la obesidad infantil (Pérez-Herrera \& Cruz-López, 2019), problemade sal ud pública en el que interfieren factores sociales, al imenticiosy sedentarismo.

La mayoría de los docentes encuestados expresaron haber recibido capacitación por parte de las autoridades académicas. Sin embargo, los que argumentaron que no, manifestaron la necesidad de capacitaciones de interés en relación con el desarrollo de competencias pedagógicas con TIC, trabajo con padres, madres y tutores legales y en lo referente al protocolo de actuación cuando se regrese a clases presenciales. Es fundamental la formación continua del profesorado de EF, desde una perspectiva crítica y reflexiva en donde se compartan experiencias, teniendo la finalidad de mejora docente (Cañabate, Tesouro, Puigggali \& Zagalaz, 2019).

En este sentido, diversos autores han hecho reco- mendaciones al profesorado de EF, con la finalidad de solventar de la mejor manera posible la situación actual. Para Posso, Otañez, Paz, O rtiz y Núñez (2020), con la utilización TIC, la EF se encuentra en camino de descubrir metodologías y estrategias didácticas para tener un mayor impacto en la población; sin embargo, se requiere el apoyo de instancias gubernamentales que doten el equipamiento necesario.

Durante la etapa de confinamiento, se recomienda al profesorado de EF adaptar y contextualizar activida des tomando en cuental las particularidades de los al umnos y los recursos materiales a su disposición, mismos que deben ser ergonómicos; también, estimular la expresión corporal por medio de dramatizaciones de películas involucrando a los integrantes de la familia (Burgueño et al., 2021). Ante un eventual regreso a clases presenciales, el COLEF ha recomendado estar actualizado con las normativas gubernamentales y del centro escolar, realizar planificaciones didácticas tomando en cuenta contenidos indispensables, los aprendizajes no al canzados y adaptaciones para alumnos con necesidades educativas especiales. En cuanto al espacio, priorizar actividades sin contacto físico, con distancia miento de no menos de 1.5 metros y dar prioridad a utilizar espacios al aire libre. En referencia a los materiales didácticos, enfocar actividades con poca o nula necesidad de material, utilizar instrumentos fáciles de desinfectar, evitar el contacto con superficies, no compartir el material y permitir que el alumno diseñe su propio material. En cuanto al tamaño de los grupos, se recomienda gestionar con las autoridades la reducción del número de alumnos, así como, organizar subgrupos (COLEF, 2020).

\section{Conclusión}

El profesorado de EF que participó en este estudio enfrentó diversos retos para poder desarrollar su traba jo: problemas conexión a internet, falta de comunica ción y/ 0 disposición de los padres, madres o tutores legales. Adaptación de clases al material en casa de los estudiantes, la eval uación fue compleja y de poca credibilidad; sin embargo, el desarrollo de actitudes, valores y habilidades en favor del aprendizaje fue fundamental para realizar su trabajo. Para los profesores, el confina miento colocó a sus estudiantes en riesgo de sufrir afectaciones psicológicas relacionadas con sociabilidad, adquisición de malos hábitos alimenticios y sedentarismo. Es prudente destacar la inquietud de al gunos profesores respecto a los lineamientos de actuación en el retorno a 
las sesiones presenciales.

Este estudio no estuvo exento de limitaciones, dado su carácter descriptivo, el reducido número de la muestra no permite generalizar la manera en que el profesorado de EF impartió sus sesiones durante el periodo analizado. Así mismo, el instrumento contempló preguntas con opción de respuesta abierta, en al gunos casos las respuestas fueron limitadas o de nula información para analizar, fue un estudio mayormente cualitativo; en este sentido, los resultados fueron producto de la interpretación de los investigadores; no obstante, se considera que se encontró información valiosa en torno a la COVID-19 y sus repercusiones en la docencia de EF. En función de los resultados obtenidos y las limita ciones, es posible sugerir líneas de actuación futuras. La primera, realizar estudios con mayor número de participantes e inclusive analizando diferentes contextos demográficos que permitan generalizar, confirmar y discutir resultados. La segunda, analizar la actualización docente respecto a las medidas de seguridad y estrategias didácticas para impartir clases de EF en etapas de desescalada y en el eventual regreso a clases presenciales.

\section{Referencias}

Adhikari, S. P., Meng, S., Wu,Y. J., M ao,Y. P.,Ye, R. X., Wang, Q.Z., $\ldots \&$ Zhou, H. (2020). Epidemiology, causes, clinical manifestation and diagnosis, prevention and control of coronavirus disease (COVID-19) during the early outbreak period:A scoping review. Infectious D iseases of Poverty, 9(1), 1-12. doi: 10.1186/ s40249020-00646-x

Aznar, I., Cáceres, M. P., Trujillo, J. M ., \& Romero, J. M. (2019). Impacto delasappsmóvilesen laactividad física: un meta-análisis. Retos: nuevas tendencias en educación física, deportey recreación, (36), 52-57. doi: 10.47197/ retos. v36i36.66628

Baena-M orales, S., López-M orales, J., \& García-Taibo, O. (2020). La intervención docente en educación física durante el periodo de cuarentena por COVID-19 (Teaching intervention in physical education during quarantine for COVID-19). Retos nuevastendencias en educación física, deporte y recreación, (39), 388-395. doi: 10.47197/ retos. v0i39.80089

Baños, R., \&Arrayales, E. (2020). Predicción del aburrimiento en la educación física a partir del climamotivacional. Retos: nuevastendencias en educación física, deporte y recreación, (38), 83-88. doi: $10.47197 /$ retos. v38i38.74301

Baños, R., M arentes-Castillo, M., Zamarripa, J., Baena-Extremera, A., O rtiz-Camacho, M., \& Duarte-Félix, H. (2019). Satisfacción, aburrimiento e importancia de la educación física en la intención de realizar actividad física extraescolar en adolescentes mexicanos. Cuadernos de Psicología del Deporte, 19(3), 205-215. doi: 10.6018/ cpd. 358461

Barahona, J. D., García, J. M., \& Pañego, M. M. (2019). Estudio de lasactitudesy el interés delosdocentes de primariadeeducación física por lasT IC en laComunidadValenciana. Retos: nuevastendencias en educación física, deporte y recreación, (35), 267-272. doi:
10.47197/ retos. v0i35. 63355

Barrientos, E., López,V., \& Pérez-Brunicardi, D. (2019). ¿Por qué hago evaluación formativay compartiday/ o eval uación parael aprendizaje en EF? Lainfluenciadelaformación inicial y permanente del profesorado. Retos: nuevas tendencias en educación física, deporte y recreación, (36), 37-43. doi: 10.47197/ retos. v36i36.66478

Bermejo, J., Pulido, D., Galmes-Panades, A. M., Serra, P.Vidal, J., \& Ponseti, F.J. (2021). Educación física y universidad: Evaluación deunaexperienciadocenteatravés del aprendizaje cooperativo. Retos: nuevas tendenciasen educación física, deportey recreación, (39), 20.

Betancourt, M., Bernate, J., Fonseca, I., \& Rodríguez, L. (2020). Revisión documental de estrategias pedagógicas utilizadas en el áreade laeducación física, parafortalecer las competencias ciudadanas. Retos: nuevastendenciasen educación física, deportey recreación, (38), 845-851. doi: 10.47197/ retos. v38i38. 74918

Blocken, B., Malizia, F., Van Druenen,T., \& Marchal,T. (2020).Towards aerodynamically equival ent COVID-191.5 m social distancing for walking and running. Urban Physics, W ind Engineering \& SportsAerodynamics. Recuperado dehttps: / / bit. ly/ 32] wZzU Bogantes, C., M onge, M., González, E., Víquez, G. \& Vargas, G. (2020). Sobrepeso, obesidad, niveles de actividad física y autoestimade laniñez centroamericana: un análisiscomparativo entre países. Retos: nuevastendencias en educación físca, deportey re creación, (37), 238-246. doi: 10.47197/ retos. v37i37.71680

Burgueño, R., Espejo, R., López-Fernández, I. \& \& Gil-Espinosa, F.J. (2020). Educación Física de calidad en casa paraniños: unapropuestade aplicación curricular en Educación Primaria. Journal of Sport and H ealth Research. 12 (2): 270-287.

Burgueño, R., Bonet-M edina, A., Cerván-Cantón, A., Espejo, R., Fernández-Berguillo, F., Gordo-Ruiz, F., Linares-Martínez, H., ... \& Gil-Espinosa, F. (2021). Educación Físicaen Casa deCalidad. Propuestade aplicación curricular en Educación Secundaria O bligatoria. Retos: nuevastendencias en educación física, deportey re creación, (39), 787-793.

Calatayud, M. (2019). Unaoportunidad paraavanzar haciala evaluación auténtica en Educación Física. Retos: nuevastendenciasen educación física, deporte y recreación, (36), 259-265. doi: 10.47197/ retos. v36i36. 67540

Cañabate, D., Tesouro, M ., Puigggali, J. \& \& Zagalaz, M. (2019). Estado actual de la Educación Física desde el punto de vista del profesorado. Propuestas de mejora. Retos: nuevastendenciasen educación física, deporte y recreación, (35), 47-53. doi: 10.47197/ retos. V0i35.63038

Canales-N úñez, P., Aravena, O ., Carcamo-0 yarzún, J., Lorca, J., \& Martinez-Salazar, C. (2018). Prácticas pedagógicas que favorecen u obstaculizan lainclusión educativa en el aula de educación físicadesdela per spectivadel alumnado y profesorado. Retos: nue vastendencias en educación física, deportey recreación, (34), 212-217. doi: 10.47197/ retos. voi34.59620

Carriedo,A., M éndez-Giménez,A., Fernández-Río,J., \& Cecchini,J. A. (2020). N uevas posibilidades y recur sos paralaenseñanzade la expresión corporal en educación física: internet y los retos virales. Retos: nuevastendencias en educación física, deportey recreación, 37(37), 722-730. doi: 0.47197/ retos.v37i37.69147

Celis-Morales, C., Salas-Bravo, C.,Yáñez, A., \& Castillo, M. (2020). Inactividad físicay sedentarismo. La otra cara de los efectossecundarios de laPandemiadeCOVID-19. Revista médica deChile, 148(6), 885-886. doi:10.4067/ S0034-98872020000600885

Chen, P., Mao, L., Nassis, G. P., Harmer, P., Ainsworth, B. E., \& Li,F. 
(2020a).W uhan coronavirus (2019-nCoV):Theneed tomaintain regular physical activity whiletaking precautions. Journal of Sport and Health Science 9(2), 103-104. doi: 10.1016/ j.jshs. 2020.02.001

Chen, P., Mao, L., Nassis, G. P., Harmer, P., Ainsworth, B. E. , \& Li, F. (2020b). Returning Chinese school-aged children and adolescents to physical activity in the wake of COVID-19:Actions and precautions. Journal of Sport and H ealth Science, 9 (4), 322-324. doi: $10.1016 /$ j.j.jsh. 2020.04 .003

COLEF, C. (2020). Recomendaciones docentes parauna educación física escolar seguray responsable ante la «nueva normalidad». Minimización de riesgos de contagio de la COVID-19 en las clasesdeEF parael curso 2020-2021. Revista Española deEducación Física y Deportes, (429), 81-93.

Contreras, G. (2010). Diagnosing LearningAssessment Difficulties at the University Level:A Specific Casein Chile. Educación y Educadores, 13(2), 219-238. doi: 10.5294/ edu. 2010.13.2.3

Cossio-Bolaños, M. (2020). Actividad física en tiempos de cuarentenapor el COVID-19 en niñosy adolescentes. Revista Peruana de ciencia dela actividad física y del deporte, 7(2), 2-2.

DaCosta, F. C. (2006). La enseñanza de la Educación Física ante la implantación del Espacio Europeo deEducación Superior. Educación Física y deporte, 25(2), 29-43.

Declaración deH elsinki. (2015). Principioséticospara lasinvestigaciones médicas en seres humanos. Sitio web https: / / www.wma. net/ es/ policies-post/ declaracion-de-helsinki-de-la-amm-principioseticos-para-las-investigaciones-medicas-en-seres-humanos/

Díaz, J. (2020). Retos y oportunidades de la tecnología móvil en la educación física. Retos: nuevastendencias en educacion fisica, deportey recreacion, 37(37), 763-773. doi: 10.47197/ retos. v37i37.68851

Dixit, S. (2020). Can moderateintensity aerobic exercise bean effective and valuable therapy in preventing and controlling the pandemic of COVID-19? M edical Hypotheses, 143, 109854. doi: 10.1016/ j. mehy.2020.109854

Escobar-Pérez, J., \& Cuervo-Martínez, Á. (2008).Validez decontenido y juicio de expertos: unaaproximación asu utilización. Avances en medición, 6(1), 27-36.

Fernández, J. M. , M ontenegro, M., Fernández, J., \&Tadeu, P. (2021). Impacto delasTIC en el alumnado con discapacidad en el áread Educación Física: una revisión sistemática. Retos: nuevastendencias en educación física, deportey recreación, (39), 849-856.

Fernández-Río, J. F. (2017). El Ciclo del A prendizaje Cooperativo: una guía para implementar de manera efectiva el aprendizaje cooperativo en educación física. Retos: nuevastendenciasen educación física, deporte y recreación, (32), 264-269. doi: 10.47197/ retos. v0i32. 51298

Fernández-Río, J., Calderón, A., Alcalá, D. H. , Pérez-Pueyo, Á., \& Cebamanos, M.A. (2016). Modelospedagógicos en educación física: consideraciones teórico-prácticas para docentes. Revista Española de Educación Física y D eportes, (413), 55-75.

Fuentes, J. D. (2020). Hacia una educación física pertinente en una región de altitud del Perú en épocade pandemia. Revi sta Peruana de ciencia dela actividad física y del deporte, 7(4), 2-2.

García, R. S. (2020). La reutilización lúdica del espacio urbano en tiempos delaCOVID-19. Sociología del D eporte, 1(1), 25-28. doi: 10.46661/ socioldeporte. 4999

García, S. , Sánchez, P., \& Ferriz,A. (2021). M etodologías cooperativas versuscompetitivas: efectos sobrela motivación en alumnado deEF. Retos: nuevastendencias en educacion física, deportey recreaicón, (39),65-70.

Gardner, R ., \& Stephens-Pisecco,T. (2019). Empowering educators to foster student resilience. The Clearing House. A Journal of Educational Strategies, Issues and Ideas, 92 (4-5), 125-134. doi: $10.1080 / 00098655.2019 .1621258$

Giakoni, F., Paredes, P., \& Duclos-Bastías, D. (2021). Educación Físicaen Chile: tiempo dededicación y su influenciaen lacondición física, composición corporal y nivel de actividad física en escolares. Retos: nuevastendenciasen educación física, deportey recreaicón, (39), 24-29. doi: 10.47197/ retos. v0i39.77781

Glasser, B. , (1992). Emergencev Forcing Basics of G roundedT heory Analysis. Sociology Press.

Gómez, G. , Rodríguez, C., \& Ramos, M. (2019). Larealidad virtual en el áreade educación física. Journal of Sport and H eal th Research, 11(1), 177-186.

Gómez-Gonzal vo, F., M olina-Alventosa, J. P., \& D evís, J. (2018). Los videojuegoscomo materialescurriculares: unaaproxi-mación a su uso en Educación Física. Retos: nuevas tenden-cias en educación física, deporte y recreación, (34), 305-310. doi: 10.47197/ retos. v0i34.63440

Guillamón, A., Garcia, E. \& \& Martínez, H. (2020). Influencia de un programa de actividad física sobre laatención selectivay la eficaciaatencional en escolares. Retos, nuevastendenciasen educación física, deporte y recreación, (38), 560-566. doi: 10.47197/ retos. v38i38.77191

Guillamón, A., García, E., \& M artínez, H. (2021). Ejercicio físico aeróbico y atención selectiva en escolares de educación primaria. Retos: nuevastendencias en educación física, deportey recreación, (39), 421-428.

Guthold, R., Stevens, G. A., Riley, L. M., \& Bull, F. C. (2020). Global trendsin insufficient physical activity among adolescents: A pooled analysis of 298 population-based surveys with 1.6 million participants. The Lancet Child and Adolescent H ealth, 4 (1), 23- 35. doi: 10.1016/ S2352-4642(19)30323-2

Hall, J. A., \& O choa-M artínez, P.Y. (2020). Enseñanza virtual en educación físicaen primariaen México y lapandemiapor COVID19. Revista Ciencias delaActividad Fíśca U CM ,21(2). doi: 10.29035/ rcaf. 21.2.4

Hernández, A., González, I., Sánchez,Y., \& Carrión, S. (2020. Los ambientes deaprendizajeen Educación Físicay motivación en las primeras edades. Retos: nuevastendencias en educación física, deportey recreaicón, (38), 761-767. doi: 10.47197/ retos. v38i38. 77441

Hernández, R., Fernández, C. , \& Baptista, P. (2014). M etodología de la investigación (6ta. ed.). México: M cG raw-Hill.

Islam, S. D. U., Bodrud-D oza, M., Khan, R. M., Haque, M. A., \& Mamun, M.A. (2020). Exploring COVID-19 stressand itsfactors in Bangladesh: aperception-based study. H eliyon, 6(7), e04399. doi: 10.1016/ j. heliyon.2020.e04399

Kalazich, C., Val derrama, P., Flández, J., Burboa, J., Humeres, D., Urbina, R , ... \&Valenzuela, L. (2020). O rientacionesD eportey COVID-19: Recomendaciones sobre el retorno a la actividad físicay deportes de niños niñasy adolescentes. Revista chilena de pediatría, 91(7), 75-90. doi: 10.32641/ rchped. v91i7. 2782

Martínez-Hita, F. J. (2020). Propuestas activas en Educación Física duranteel confinamiento por el Covid-19. Lecturas: Educación Física y D eportes, 25(266). doi: 10.46642/ efd. v25i266. 2178

Melnyk, Y. (2017). Monitoring of health culture formation in schoolchildren. Journal of Physical Education and Sport, 17(54), 20732079.

Moreno, J.A. , Rodríguez, C. , N avas-Parejo, M. , \& Sola, J. M. (2020). Interésy motivación del estudiantado de Educación Secundaria en el uso deAurasma en el aula de Educación Física. Retos: nuevas tendencias en educación física, deportey recreaicón, (38), 333-340. doi: 
10.47197/ retos. v38i38.76832

M uñoz-Arroyave, V. , Lavega-Burgués, P., Costes, A., Damian, S., \& Serna, F. (2020). Losjuegosmotores como recurso pedagógico para favorecer la afectividad desde la educación física. Retos: nuevastendencias en educación física, deportey recreación, (38), 166172. doi: $10.47197 /$ retos. v38i38. 76556

O rganización de las $\mathrm{N}$ aciones U nidas paralaEducación, la Cienciay laCultura. (2020). ¿Cómo estás aprendiendo durantela pandemia de CovID-19? Recuperado el 15 de enero de 2021 de https:/ / es. unesco.org/ covid19/ educationresponse

O rganización de las $\mathrm{N}$ aciones U nidas paralaEducación, la Cienciay laCultura. (2015). Carta internacional de la educación física, la actividad física y el deporte. Recuperado el 5 de noviembre de 2020 de https: / / cutt.ly/ Cur8NNQ

O rganización M undial delaSalud. (2021). COVID-19: Cronologia de la actuación de la OMS. Recuperado el 15 de enero de 2021 de https: / / www. who. int/ es/ news/ item/ 27-04-2020-whotimeline- covid-19

O rozco, J. C. (2016). Estrategias didácticasy aprendizaje de lasciencias sociales. Revista Científica de FAREM - Estelí, 5(17), 65-80. doi: 10.5377/ farem.v0i17. 2615

Pérez P, Á., \& HortigüelaA. D. (2020). ¿Y si todalainnovación no es positiva en Educación Física?: R eflexiones y consideraciones prácticas. Retos: nuevastendencias en educación física, deportey recreación, (37), 579-587. doi: 10.47197/ retos. v37i37.74176

Pérez-Herrera, A., \& Cruz-López, M. (2019). Situación actual de la obesidad infantil en M éxico. N utrición H ospitalaria, 36(2), 463469. doi: 10.20960/ nh.2116

Piedra, J. (2020). Redes socialesen tiempos del COVID-19: el caso de laactividad física. Sociología del D eporte, 1(1), 41-43. doi: 10.46661/ socioldeporte. 4998

Pinasa,V. G. (2020). COVID-19: Lacrisisha afectado atodos. Revista Española deEducación Física y D eportes, (429), 15-18.

Posso, R. J. , 0 tañez, J. M., Paz, S. , O rtiz, N. A. \& \& Núñez, L. F. X. (2020). Por unaEducación Físicavirtual en tiemposdeC OVID. Podium. Revista de Ciencia yTecnología en la Cultura Física, 15(3), 705-716.

Recio, D. (2017).Video-leccionesy evaluación formativa: unaprácticaen educación física. Revista Infancia, Educación yAprendizaje,3(2), 272-278.

Rodríguez, A. C., Valenzuela, A.V., \& Martínez, B. J. S.A. (2016). La importanciadelaeducación física en el sistemaeducativo. EmásF: revista digital deeducación física, (43), 83-96.

Román, F., Fores, A., Calandri, I., Gautreaux, R., Antúnez, A., O rdehi, D., ... \&Allegri, R. (2020). Resiliencia en docentes en distanciamiento social preventivo obligatorio durantelapandemia deCOVID-19. JONED. Journal of N euroeducation, 1(1), 76-87. doi: 10.1344/ joned. v1i1.31727

Sal azar, C. M ., \& G astélum, G. (2020). Teoría de la autodeterminación en el contexto de educación física: U narevisión sistemática. Retos: nuevastendenciasen educación física, deportey recreación, (38), 838-844. doi: 10.47197/ retos. v38i38. 72729

SecretariadeEducación Pública. (2020). Bol etín N 0. 118 N o separalizó el Sistema Educativo ante la pandemia de COVID -19; regresará a clases fortalecido: Esteban M octezuma Barragán. Recuperado el 15 deenero de 2021 de https:/ / www. gob. mx/ sep/ es/ articulos/ boletinno-118-no-se-paral izo-el-sistema-educativo-ante-la-pandemia de-covid-19-regresara-a-clases-fortalecido-esteban-moctezumabarragan?idiom=es\# : : text=El\%20Secretario\%20de\%20Edu caci \% C 3\% B 3 n \% 20P\% C 3\% B A blica, de \% 201 a \%20educaci\%C3\%B3n\%20en\%20M \%C3\%A9xico.
Shechter, A., Diaz, F., M oise, N. , Anstey, D. E. ,Ye, S., Agarwal, S. , ... \& Claassen, J. (2020). Psychological distress, coping behaviors, and preferencesfor support among $N$ ew York healthcareworkers during the COVID-19 pandemic. General hospital psychiatry, 66, 1-8. doi: 10.1016/ j. genhosppsych. 2020.06.007

Silva-Filho, E., Sales, A. L., Da Silva, J. R. D., DaSilva, D. , Andrade, R., \& Andrade, J. D. (2020). Physical education role during coronavirus disease 2019 (COVID-19) pandemic Physical education and COVID-19. M otriz:Revista deEducação Física, 26(2). / doi: 10.1590/ s1980-6574202000020086

Soares, W., Grava, M. , Cassan, S., Navarro, D., Roque, T., Soares, G., $\&$ Lopes, E. (2021). Razones por las que los estudiantes de secundaria abandonan las clases de educación física. Retos: nuevas tendencias en educación física, deportey recreación, (39), 705-712.

Taylor, S., Landry, C. A., Paluszek, M. M., Fergus, T. A., M cKay, D., \&Asmundson, G. J. (2020). COVID stresssyndrome: Concept, structure, and correlates. Depression and Anxiety, 37 (8), 706-714. doi: 10.1002/ da. 23071

Vera, I., \& Gabari, I. (2019). La resilienciacomo factor protector del estréscrónico en docentes. European J ournal of Investigation in $\mathrm{H}$ ealth, Psychology and Education 9(3), 159-175.

Villaverde-Caramés, E., Fernández-Villarino, M .A. , Toja-Reboredo, B. \& González-Valeiro M.A. (2021). Revisión de la literatura sobre las característicasquedefinen a un buen docente de Educación Física: consideracionesdesdelaformación del professorado. Retos: nuevastendenciasen educación física, deportey recreación, (41), 471-479. doi: 10.47197/ retos. v0i41.84421

Wang, G. , Zhang, Y. , Zhao, J., Zhang, J . \& J jiang, F. (2020). Mitigate the effects of home confinement on children during the COVID19 outbreak. The Lancet, 395(10228), 945-947. doi: 10.1016/ S0140-6736(20)30547-X

Zueck, M. D. C., Ramírez, A.A., Rodriguez, J. M., \& Irigoyen, H. E. (2020). Satisfacción en las clases de Educación Física y la intencional idad de ser activo en niños del nivel de primaria. Retos: nuevastendenciasen educación física, deportey recreación, (37), 33-40. doi: $10.47197 /$ retos. v37i37.69027

\section{Anexo}

\section{Cuestionario experiencias docentes del profesorado de EF durante el} confinamiento por COVID-19

A preciable profesor de educación física, este cuestionario tiene la finalidad de conocer sus experiencias respecto al impartir clases de EF durante el confinamiento producto de la Pandemia por COVID -19. Agradecemos su colaboración para contestar las siguientes preguntas. Es importante destacar que sus respuestas son anónimas y se util izarán únicamente para fines de investigación.

Datos generales:

Género: Masculino/ Femenino

Edad:

Nivel en el que imparte clases: Preescolar/ primaria/ secundaria

Ubicación de la escuela: rural / urbana

Años de experiencia impartiendo clases

Lea con detenimiento cada una de las siguientes preguntas y sea abundante es sus respuestas

1. iHa logrado cumplir con el programa de Educación Física de la Secretaría de Educación Pública durante el confinamiento por COVID-19? Sí/no

2. ¿Q ué estrategias didácticas ha utilizado para impartir su clase durante el confinamiento por COVID-19?

3. ¿Se siente satisfecho con el dominio de dichas estrategias didácticas? Sí/ no

4. En caso de que la respuesta a la pregunta anterior sea negativa, por favor explique su respuesta.

5. ¿Q ué criterios de evaluación ha utilizado para conocer los avances de sus estudiantes durante el confinamiento por COVID-19?

6. ¿Cómo considera el impacto de una evaluación a distancia a sus alumnos? Por favor explique su respuesta anterior.

7. ¿Ha encontrado al guna dificultad para desarrollar su trabajo docente? Sí/ no

8. En caso de que respuesta anterior sea afirmativa, por favor explique su respuesta

9. ¿Q ué habilidades, actitudes o valores ha tenido que desarrollar para impartir sus clases en esta etapa de confinamiento?

10. ¿Considera que la actual situación de confinamiento pudiera afectar en alguna medida la salud de sus estudiantes? Sí/ no

11. En caso de que su respuesta a la pregunta anterior sea afirmativa iDe qué manera considera que la actual situación de confinamiento pudiera afectar en alguna medida la salud de sus estudiantes?

12. ¿Ha recibido capacitación por parte de las autoridades educativas para darle a usted herramientas necesarias para afrontar el reto de la pandemia por COVID-19? Sí/ no

13. En caso de que si respuesta anterior fuera negativa ¿Q ué capacitaciones requiere? 